\title{
Merkel cell carcinoma expresses vasculogenic mimicry: demonstration in patients and experimental manipulation in xenografts
}

\author{
Cecilia Lezcano ${ }^{1}$, Sonja Kleffel ${ }^{2,3}$, Nayoung Lee ${ }^{2,3}$, Allison R Larson²,3, Qian Zhan ${ }^{3,4}$, Andrew DoRosario ${ }^{3,5}$, \\ Linda C Wang ${ }^{6}$, Tobias Schatton ${ }^{2,3,7}$ and George F Murphy $y^{3,4}$
}

\begin{abstract}
Merkel cell carcinoma (MCC) is a highly virulent cutaneous neoplasm that, like melanoma, is a frequent cause of patient morbidity and mortality. The cellular mechanisms responsible for the aggressive behavior of MCC remain unknown. Vasculogenic mimicry (VM) is a phenomenon associated with cancer virulence, including in melanoma, whereby anastomosing laminin networks form in association with tumor cells that express certain endothelial genes. To determine whether VM is a factor in MCC, we employed a relevant xenograft model using two independent human MCC lines. Experimentally induced tumors were remarkably similar histologically to patient MCC, and both contained laminin networks associated with vascular endothelial-cadherin (CD144) and vascular endothelial growth factor receptor 1, as well as Nodal expression typical of VM in melanoma. Moreover, two established chemotherapeutic agents utilized for human MCC, etoposide and carboplatin, induced necrosis in xenografts on systemic administration while enriching for laminin networks in apparently resistant viable tumor regions that persisted. These findings for the first time establish VM-like laminin networks as a biomarker in MCC, demonstrate the experimental utility of the MCC xenograft model, and suggest that VM-rich regions of MCC may be refractory to conventional chemotherapeutic agents.

Laboratory Investigation (2014) 94, 1092-1102; doi:10.1038/labinvest.2014.99; published online 11 August 2014
\end{abstract}

Merkel cell carcinoma (MCC) is a rare and highly aggressive cutaneous neoplasm with a high rate of morbidity and mortality. ${ }^{1,2}$ The mechanisms underlying MCC aggressiveness have not been fully characterized. However, like melanoma, MCC was found to express virulence-conferring factors, such as the embryonic neural crest stem cell transcription factor, SRY (sexdetermining region $\mathrm{Y}$ )-box 2 (SOX2), which is associated with invasiveness and tumorigenesis in melanoma. ${ }^{3}$ An additional biomarker and potential mechanism associated with tumor aggressiveness is vasculogenic mimicry (VM), whereby anastomosing periodic acid-Schiff (PAS)- and laminin-positive networks develop within tumors. ${ }^{4,5}$ In melanoma, vascular endothelial growth factor receptor 1 (VEGFR-1) ${ }^{+}$tumor subsets drive tumor growth and form patterned networks with structural and antigenic characteristics of VM. Indeed, pioneering works by Hendrix, Folberg, and others have shown these laminin networks to be associated with melanoma virulence by forming channels that facilitate perfusion via direct or indirect connections with authentic vessels. ${ }^{6,7}$ Whereas tumor angiogenesis involves ingrowth and sprouting of stromal vessels lined by platelet endothelial cell adhesion molecule 1 (CD31)-expressing endothelial cells, ${ }^{8-10} \mathrm{VM}$ networks are intimately associated with tumor cells that express endothelial genes encoding for vascular endothelial-cadherin (CD144), tyrosine kinase with immunoglobulin-like and EGF-like domain 1 (TIE-1), and VEGFR-1, but not CD31. 5,6,11,12 In this study, we aimed to assess whether MCC similarly harnesses VM to propagate tumor aggressiveness. Using patient biopsies and a xenograft model relevant to human disease, here we show that VM may be documented clinically and experimentally manipulated in MCC, establishing VM as a novel biomarker for this important tumor type.

\footnotetext{
'Department of Pathology, University of Pittsburgh Medical Center, Pittsburgh, PA, USA; ${ }^{2}$ Department of Dermatology, Brigham and Women's Hospital, Boston, MA, USA; ${ }^{3}$ Harvard Medical School, Boston, MA, USA; ${ }^{4}$ Department of Pathology, Brigham and Women's Hospital, Boston, MA, USA; ${ }^{5}$ Center for Cutaneous Oncology, DanaFarber/Brigham and Women's Cancer Center, Boston, MA, USA; ${ }^{6}$ Institute for Cancer Care, Mercy Medical Center, Baltimore, MD, USA and ${ }^{7}$ Transplantation Research Center, Children's Hospital, Boston, MA, USA

Correspondence: Dr GF Murphy, Department of Pathology, Brigham and Women's Hospital, Harvard Medical School, 221 Longwood Avenue, EBRC Suite 401, Boston, MA 02115, USA.

E-mail: gmurphy@rics.bwh.harvard.edu

Received 13 March 2014; revised 24 June 2014; accepted 27 June 2014
} 


\section{MATERIALS AND METHODS}

\section{Cell Lines and Cell Culture}

Authenticated human MCC cell lines (MKL-1 and WaGa) were obtained courtesy of Dr James DeCaprio at the Dana-Farber Cancer Institute, Boston, $\mathrm{MA},{ }^{13}$ and were cultured $<6$ months in RPMI 1640 medium supplemented with 20\% (v/v) FBS and $1 \%$ (v/v) penicillin/streptomycin (Gibco, Life Technologies, Grand Island, NY). Authenticated human umbilical vein endothelial cells (HUVEC) were obtained from the American Type Culture Collection and cultured in M199 medium supplemented with $10 \%(\mathrm{v} / \mathrm{v})$ FBS, $1 \%(\mathrm{v} / \mathrm{v})$ penicillin/ streptomycin (Gibco, Life Technologies), $100 \mu \mathrm{g} / \mathrm{ml}$ (wt/v) endothelial cell growth supplement (Biomedical Technologies, Ward Hill, MA), $100 \mu \mathrm{g} / \mathrm{ml}$ (wt/v) heparin, $100 \mathrm{nM} \mathrm{(v/v)}$ hydrocortisone, and $100 \mathrm{nM}(\mathrm{v} / \mathrm{v})$ ascorbic acid in fibronectincoated $(20 \mu \mathrm{g} / \mathrm{ml})$ flasks (Sigma-Aldrich, St Louis, MO).

\section{Generation of Drug-Resistant MCC lines}

MKL-1 and WaGa cells were incubated in growth media as above supplemented with weekly increasing doses of carboplatin ( $\leq 150 \mu \mathrm{M}$, Sigma) or etoposide ( $\leq 3 \mu \mathrm{M}$, Sigma) over the course of 2 months. CD144, LAMA3, LAMB3, and LAMC2 mRNA expressions were quantified as described below.

\section{RNA Extraction, Reverse Transcription, and Real-Time Quantitative PCR}

Total RNA was isolated from vehicle-treated, carboplatin- or etoposide-resistant MKL-1 and WaGa cells, and HUVEC using the RNeasy Plus Mini Kit (Qiagen, Venlo, Limburg). Standard cDNA synthesis reactions were carried out using the SuperScript VILO cDNA synthesis kit (Invitrogen, Life Technologies) and reverse transcribed products were amplified with the Fast SYBR Green Master Mix (Applied Biosystems, Life Technologies) according to the manufacturer's instructions. The primers for detection of human CD144 by real-time quantitative RT-PCR were: 5'-CAGCCCAAAGTG TGTGAGAA- $3^{\prime}$ (forward) and $5^{\prime}$-CGGTCAAACTGCCCATA CTT-3' (reverse), for human LAMA3 detection: 5'-ATCTGG AGTCGAAGTCCGACTG-3' (forward) and 5'-TTGTAGACA CAGGTGAGCTGGC-3' (reverse), for human LAMB3 detection: $5^{\prime}$-ACCACACCGAAGGCAAGAAC- $3^{\prime}$ (forward) and 5'-GGTTGGCGTAGGTGAGTCCA-3' (reverse), for human LAMC2 detection: $5^{\prime}$-AGGCTGTCCAACGAAATGGG-3' (forward) and $5^{\prime}$-GGAGCTGTGATCCGTAGACCA- $3^{\prime}$ (reverse), and for human $18 \mathrm{~S}$ rRNA detection: $5^{\prime}$-GATGGGCGGCGGA AAATAG- $3^{\prime}$ (forward) and $5^{\prime}$-GCGTGGATTCTGCATAATG GT-3' (reverse). Kinetic PCR was performed on a StepOne Plus Real-Time PCR System (Applied Biosystems). All samples were run in triplicate. The relative amounts of PD-1 transcripts were analyzed by the $2\left(-\Delta \Delta C_{\mathrm{t}}\right)$ method as described previously. ${ }^{14}$

\section{Animals}

Non-obese diabetic/severe combined immunodeficiency interleukin-2 $\mathrm{Rg}^{-1-}$ knockout (NSG) mice were purchased from The Jackson Laboratory (Bar Harbor, ME). Mice were maintained in accordance with the institutional guidelines of Harvard Medical School and experiments were performed according to approved experimental protocols.

\section{Human MCC Xenotransplantation and Carboplatin and Etoposide Treatment}

For tumorigenicity studies, MKL-1 or WaGa MCC cells were injected subcutaneously into the bilateral flanks of recipient NSG mice $\left(1 \times 10^{7} /\right.$ injection $)$ as described. ${ }^{15}$ Tumorigenic growth was assayed after 6 weeks of growth, unless protocolstipulated euthanasia necessitated sacrifice before this, in situations of excessive tumor growth or animal morbidity. At day 34 post tumor cell inoculation, mice were randomized to carboplatin, etoposide, or vehicle control treatment groups with similar tumor volumes. Carboplatin (Novaplus, Lake Forest, IL) or etoposide (APP pharmaceuticals, Schaumburg, IL) was administered daily by intraperitoneal injection for 6 consecutive days, at $75 \mathrm{mg} / \mathrm{kg}$ or $10 \mathrm{mg} /$ $\mathrm{kg}$ body weight, respectively, and control animals were given vehicle only, PBS, at equal volumes as previously described. ${ }^{16}$ Tumor volumes were measured daily for the duration of the treatment, xenografts collected 1 day following administration of the final treatment dose, and frozen or paraffinembedded MCC sections were prepared for subsequent immunohistochemical analysis.

\section{Human MCC Samples}

According to IRB-approved protocols, seven clinically annotated formalin-fixed paraffin-embedded (FFPE) specimens of MCC were obtained from six patients; four of them were cutaneous lesions (of which two were primary lesions and two were recurrent cutaneous lesions) and three were lymph node metastases. The two specimens that corresponded to cutaneous recurrences were obtained after the patients were treated with at least one cycle of the combination of carboplatin and etoposide.

\section{Histochemistry, Immunohistochemistry, and Immunofluorescence}

All patient MCCs $(n=7)$ and xenografts $(n=41 ; 6 \mathrm{WaGa}$ vehicle, $7 \mathrm{MKL}-1$ vehicle, $8 \mathrm{WaGa}$ etoposide, $8 \mathrm{WaGa}$ carboplatin, $6 \mathrm{MKL}-1$ etoposide, and $6 \mathrm{MKL}-1$ carboplatin) were stained with hematoxylin and eosin (H\&E). Biomarkers of proven relevance in the detection of VM were selected (Table 1) and employed for immunohistochemistry (IHC). All patient specimens and selected xenografts $(n=18 ; 3$ WaGa vehicle, 3 MKL-1 vehicle, $3 \mathrm{WaGa}$ etoposide, $3 \mathrm{WaGa}$ carboplatin, 3 MKL-1 etoposide, and 3 MKL-1 carboplatin) were stained for PAS, laminin (Dako, Carpinteria, CA), Ulex europaeus-I (Sigma-Aldrich), and human or mouse CD31 (Bethyl Laboratories, Montgomery, TX, or Abcam, Cambridge, MA, respectively; used on human MCC or xenograft sections, respectively) in FFPE tissue. None of the anti-CD31 antibodies employed is species specific, and 
Table 1 Biomarkers employed for identification of VM vs true angiogenesis

\begin{tabular}{|c|c|c|c|}
\hline Marker & VM & Angiogenesis & Reference \\
\hline \multirow[t]{2}{*}{ CD31 } & - & Endothelium & Hendrix et $a l^{12}$ \\
\hline & & & Folberg et al ${ }^{47}$ \\
\hline \multirow[t]{2}{*}{ CD144 } & Tumor cells & Endothelium & Hendrix et $a l^{12}$ \\
\hline & & & Frank et $a l^{5}$ \\
\hline \multirow[t]{3}{*}{ VEGFR-1 } & Tumor cells & Endothelium & Shibuya ${ }^{48}$ \\
\hline & & & Vartanian et a ${ }^{49}$ \\
\hline & & & Frank et $a l^{5}$ \\
\hline \multirow[t]{2}{*}{ Nodal } & Tumor cells & - & McAllister et al ${ }^{17}$ \\
\hline & & & Hendrix et $a^{50}$ \\
\hline \multirow[t]{2}{*}{ Laminin } & BM & BM & Seftor et $a^{51}$ \\
\hline & & & Simon-Assmann et al ${ }^{52}$ \\
\hline \multirow[t]{2}{*}{ PAS } & $\mathrm{BM}$ & BM & Folberg et $a^{47}$ \\
\hline & & & Maniotis et $a^{6}$ \\
\hline
\end{tabular}

Abbreviations: BM, basement membrane; CD31, platelet endothelial cell adhesion molecule 1; CD144, vascular endothelial-cadherin; PAS, periodic acid-Schiff; VEGFR-1, vascular endothelial growth factor receptor 1; $\mathrm{VM}$, vasculogenic mimicry.

human-mouse cross-reactivity was anticipated and encountered. FFPE patient tissue $(n=4)$ was also stained for CD144 (Cell Signaling, Danvers, MA). FFPE samples were deparaffinized and epitope retrieval was achieved by enzymatic digestion with proteinase $\mathrm{K}$ (New England BioLabs, Ipswich, MA) for laminin detection or by heating tissue sections in sodium citrate solution ( $\mathrm{pH} \mathrm{6.0;} \mathrm{Dako)} \mathrm{for} \mathrm{hu-}$ man and mouse CD31 as well as for CD144. Frozen sections from xenografts $(n=6 ; 1 \mathrm{WaGa}$ vehicle, $1 \mathrm{MKL}-1$ vehicle, 1 WaGa etoposide, 1 WaGa carboplatin, 1 MKL-1 etoposide, and 1 MKL-1 carboplatin) were utilized for CD144 (Cell Signaling), Nodal (Abnova, Golden, CO), and VEGFR-1 (R\&D Systems, Minneapolis, MN) IHC. All sections were incubated overnight with primary antibodies at room temperature $\left(\sim 25^{\circ} \mathrm{C}\right)$ followed by 2-h incubation with horseradish peroxidase (HRP)-conjugated goat anti-rabbit (for Ulex, human and mouse CD31, CD144, and laminin), horse anti-goat (for VEGFR-1), and horse anti-mouse (for Nodal) secondary antibodies (all Vector Laboratories, Burlingame, CA) at room temperature. HRP substrate NovaRed (Vector Laboratories) was used for immunoreactivity detection. Double labeling for human CD31(AbD Serotec, Kidlington, UK)-CD144 (Cell Signaling) was performed as described above, incubating a tissue sample with Alexa Fluor 488 and 594 goat anti-mouse and goat anti-rabbit secondary antibodies (Invitrogen), respectively. Double labeling for laminin and associated VM markers was not utilized, as the former requires proteinase $\mathrm{K}$ digestion that abrogates bioreactivity for the second epitopes. Adjacent sections were used for comparative purposes of single epitope expression to minimize likelihood of variation based in section depth (all sections were 4-6 $\mu \mathrm{m}$ thick).

In addition, lungs from 20 mice ( 3 WaGa vehicle, 3 MKL-1 vehicle, $4 \mathrm{WaGa}$ etoposide, $4 \mathrm{WaGa}$ carboplatin, $3 \mathrm{MKL}-1$ etoposide, and 3 MKL-1 carboplatin) were stained for H\&E and cytokeratin 20 (CK20; Dako) to evaluate presence of MCC metastases in random sections.

\section{Quantitative Assessment of IHC in MCC Xenografts}

Viable areas in the periphery of xenografted tumors $(n=18$; $3 \mathrm{WaGa}$ vehicle, $3 \mathrm{MKL}-1$ vehicle, $3 \mathrm{WaGa}$ etoposide, $3 \mathrm{WaGa}$ carboplatin, 3 MKL-1 etoposide, and 3 MKL-1 carboplatin) with similar density of $\mathrm{CD} 31^{+}$vessels were sampled (two high-power $(\times 400)$ fields per specimen $)$ for computerassisted quantitative evaluation of laminin ${ }^{+}$structures consistent with VM. In addition, tumor micronodules within networks defined by CD144, Nodal, and VEGFR-1 in xenografted tumors $(n=6 ; 1 \mathrm{WaGa}$ vehicle, $1 \mathrm{MKL}-1$ vehicle, 1 WaGa etoposide, 1 WaGa carboplatin, 1 MKL-1 etoposide, and 1 MKL-1 carboplatin) were evaluated (one $\times 1000$ field) for maximal size measuring their greatest dimension. Xenografted tumors $(n=18 ; 3 \mathrm{WaGa}$ vehicle, $3 \mathrm{MKL}-1$ vehicle, 3 WaGa etoposide, $3 \mathrm{WaGa}$ carboplatin, $3 \mathrm{MKL}-1$ etoposide, and 3 MKL- 1 carboplatin) and patient MCCs $(n=7)$ were evaluated for angiogenesis by counting the number of $\mathrm{CD} 1^{+}$vessels at low magnification $(\times 100)$. Photomicrographs were taken using a Nikon Elipse 80i microscope coupled with a SPOT Insight 4.0 Mp Firewire Color Mosaic (model 14.2) camera and then analyzed employing Image J software (NIH, Bethesda, MD) for all quantitative assessments.

\section{Statistical Analysis}

Two-sided $t$-tests were used for all comparisons. A $P$-value of $<0.05$ was considered significant. Data are reported as sample means with error bars representing the s.e.m.

\section{RESULTS}

Histology of WaGa- and MKL-1-Derived MCC Xenografts

WaGa and MKL-1 cells subcutaneously injected to NSG mice both gave rise to nodular tumors composed of uniform populations of small basophilic cells with high nuclear to cytoplasmic ratios (Figure 1). The nuclei were rounded and the chromatin showed the classic finely granular, stippled appearance typical of MCC. MKL-1 tumor cells were slightly larger than WaGa cells, and MKL-1-derived tumors displayed trabecular architecture, whereas WaGa tumors grew as sheets of cells. Tumors showed a destructive relationship to subcutaneous structures, with permeation through the panniculus carnosus muscular layer. Lymphovascular invasion was not prominent, and pulmonary micrometastasis was documented in random sections in only 1 of 20 animals with up to 6 weeks of primary xenograft growth. Immunohistochemically, MCC xenografts showed CK20 positivity in 

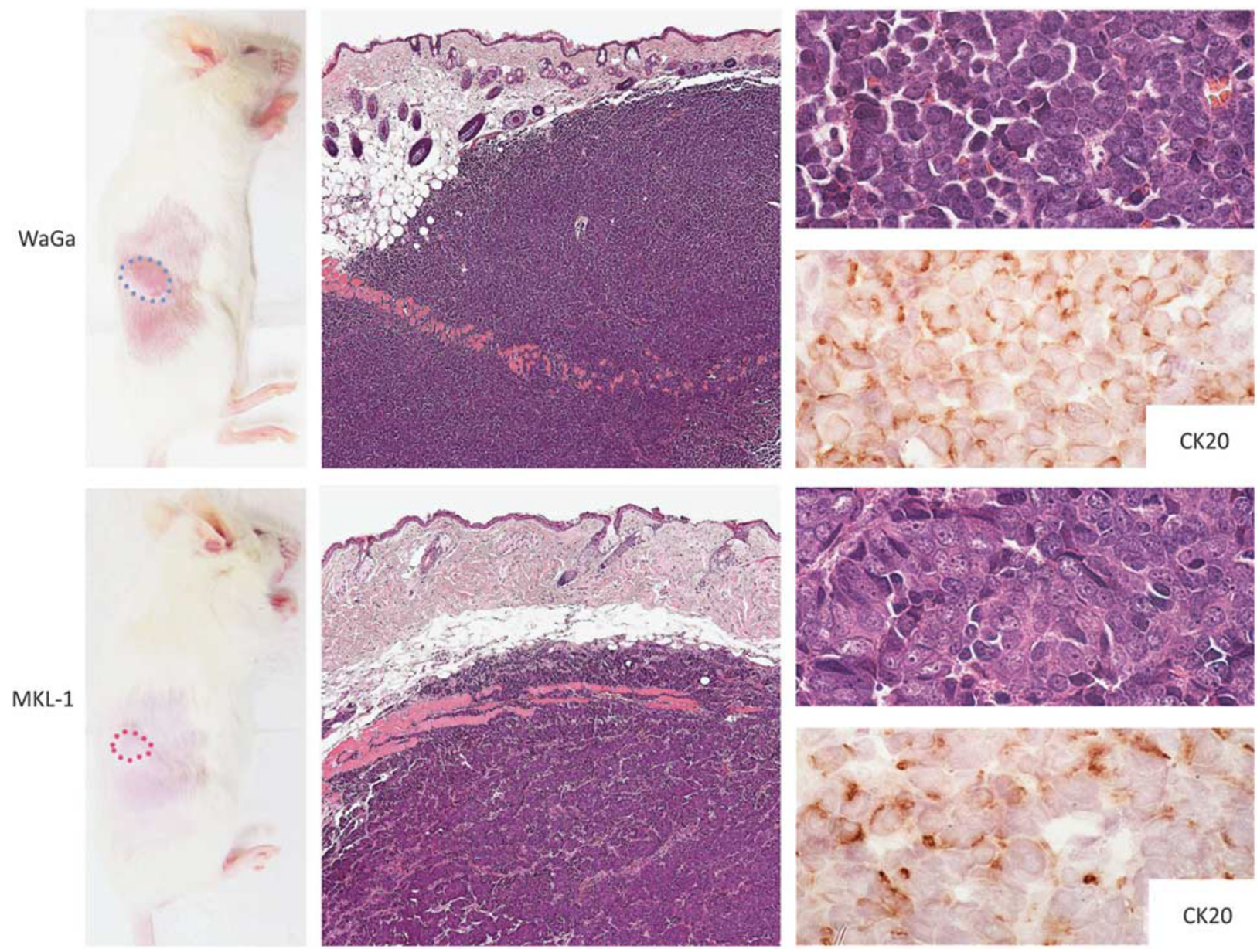

Figure 1. Merkel cell carcinoma (MCC) xenograft model. NSG mice injected with human MCC lines (WaGa, top panels; MKL-1, bottom panels) developed visible tumor masses (encircled in dotted lines) within a month (left panels; original magnification, $\times 1$ ) of tumor cell xenografting. Tumors involved the dermis and the subcutaneous tissue and were composed of uniform, small basophilic cells (center panels; original magnification, $\times 100$ ). The classic salt and pepper chromatin pattern was observed within rounded nuclei rimmed by scant cytoplasm that showed dot-like positivity for cytokeratin 20 (CK20; right panels; original magnification $\times 1000)$.

a dot-like perinuclear pattern identical to that seen in patient tumors.

$\mathrm{CD}_{3} 1^{+}$and $\mathrm{Ulex}^{+}$murine vessels were present in relatively low density throughout tumor nodules (average of 13 cross-sectional CD $31^{+}$vessel profiles per $\times 100$ field (range of 8-20); $n=18$; Figure 2). In comparison, laminin and PAS stains in adjacent sections revealed in all 18 xenografts evaluated elaborate, branching, and anastomosing networks that were in large part not spatially coincident with either $\mathrm{CD} 31^{+}$ or Ulex $^{+}$vessels, and accounted for $>90 \%$ of laminin staining. Further biomarker analysis of these networks revealed coincident staining patterns for the endothelial-associated marker CD144 (VE-cadherin), VEGFR-1, and the embryonic morphogen, Nodal, all previously associated with the phenomenon of VM in melanoma $a^{5,6,12,17}$ in representative xenografts derived from both cell lines and corresponding to each of the three treatment groups $(n=6)$. Micronodules of tumor compartmentalized by the networks defined by all of the above biomarkers (excluding CD31 and Ulex) showed a consistent maximum diameter of $40-50 \mu \mathrm{m}$, and thus the pattern of immunoreactivity for all markers corresponded both qualitatively and quantitatively.

\section{Effect of Chemotherapy on WaGa and MKL-1 Tumors}

In view of the established chemotherapy resistance of cells known to be associated with $\mathrm{VM}^{5,18}$ we next addressed WaGa- and MKL-1-derived tumors collected after systemic administration of the chemotherapeutic agents etoposide, a topoisomerase II inhibitor, or carboplatin, an alkylating-like platinum-based drug. Animals so treated exhibited xenograft tumors with centrally localized zones of prominent tumor necrosis that were not present in vehicle-treated xenografts. PAS and laminin stains showed qualitative increase over baseline in anastomosing networks in residual viable regions of tumor of both WaGa- and MKL-1-derived xenograft tumors after both modalities of chemotherapy. This increase 
was further confirmed by quantification of laminin positivity in tumor areas matched for density of $\mathrm{CD} 31^{+}$vessels (Figure 3, upper and middle panels). There was a statistically significant increase in laminin ${ }^{+}$network immunoreactivity per unit area in viable tumor areas of both MKL-1 and WaGa tumors after carboplatin, and also after etoposide in MKL-1 tumors, when compared with vehicle-treated xenografts. WaGa tumors treated with etoposide showed a trend in the same direction, although it did not reach statistical significance (Figure 3, graphs). CD144 retained a network pattern similar to that described for vehicle-treated xenografts, and qualitatively also was increased in evaluable specimens that showed augmentation in laminin networks. Quantitative analyses for other biomarkers were not performed in tissue sections obtained after chemotherapy due to technical limitations related to the extent of tumor necrosis in the samples that were allocated for frozen sectioning.

To assess further the changes after chemotherapy administration observed in vivo, we employed etoposide- and carboplatin-resistant WaGa and MKL-1 cells to compare the levels of expression of three laminin isoforms and CD144 by real-time quantitative RT-PCR against those found in vehicle-treated WaGa and MKL-1 cells (Figure 4). We found that both etoposide- and carboplatin-resistant $\mathrm{WaGa}$ and MKL-1 cells showed statistically significant increases in mRNA levels for two of the three laminin isoforms tested (LAMA3 and LAMC2), whereas only etoposide-resistant MKL-1 cells showed significantly augmented levels for the remaining laminin isoform (LAMB3). In addition, CD144 expression also was increased in etoposide- and carboplatin-resistant cell
WaGa
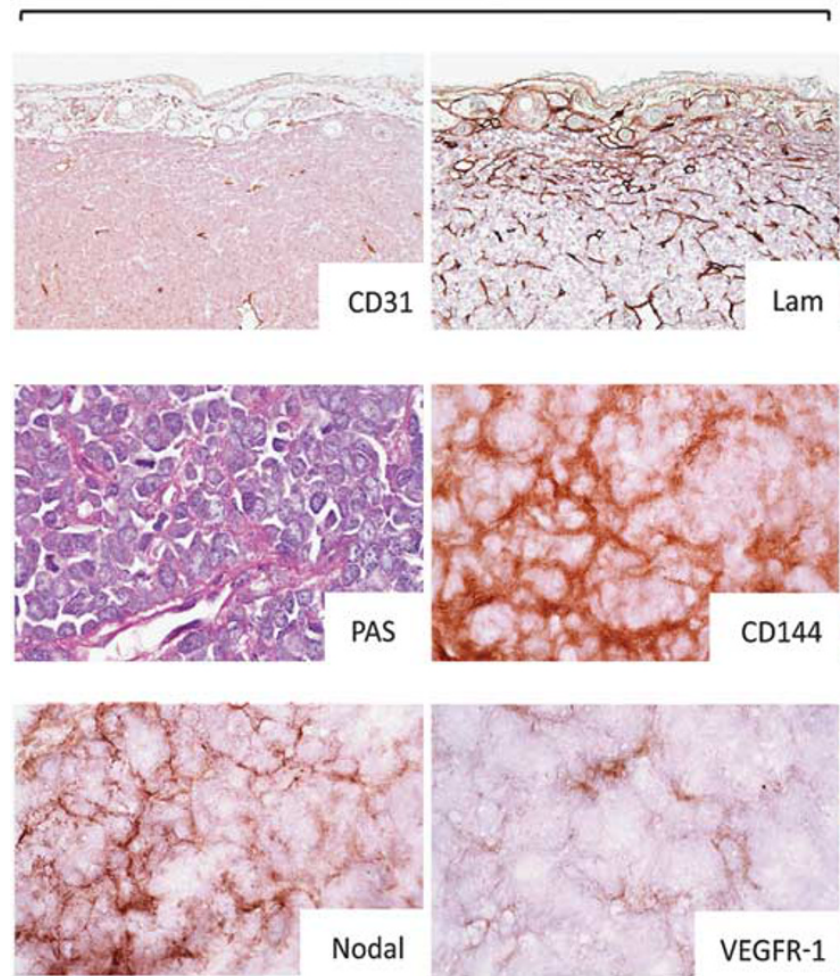

MKL-1
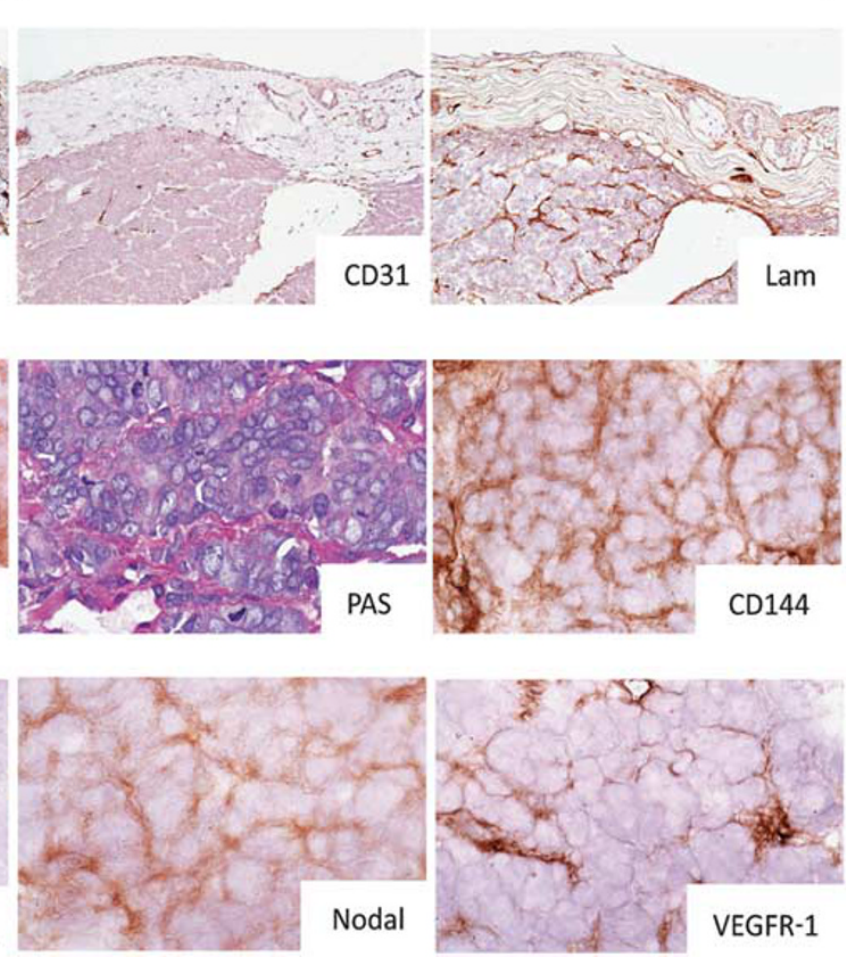

Figure 2. Vasculogenic mimicry in Merkel cell carcinoma xenografts. WaGa- and MKL-1-derived xenograft tumors contained CD31- anastomosing networks that were enhanced with PAS staining, and were associated with staining for Lam, CD144, Nodal, and VEGFR-1. Note the density and complexity of laminin ${ }^{+}$structures in comparison with CD31 $^{+}$vessels (CD31 and Lam panels; original magnification, $\times 200$; PAS, CD144, Nodal, and VEGFR-1; original magnification, $\times 1000)$. CD31, platelet endothelial cell adhesion molecule 1; CD144, vascular endothelial-cadherin; LAM, laminin; PAS, periodic acid-Schiff; VEGFR-1, vascular endothelial growth factor receptor 1.

Figure 3. Vasculogenic mimicry in Merkel cell carcinoma xenografts after chemotherapy. MKL-1 tumors treated with vehicle, carboplatin, and etoposide stained for CD31 (upper panels; original magnification, $\times 100)$ and laminin (Lam; middle panels; original magnification, $\times 100$ ); rectangular regions are representative fields enlarged for clarity (original magnification, $\times 1000$ ). Note the marked and widespread increase in laminin ${ }^{+}$networks after chemotherapy. A similar picture is observed in WaGa-derived tumors, although less diffuse (data not shown). A statistically significant increase in laminin immunoreactivity after carboplatin is observed in both cell lines, and after etoposide in MKL-1-derived tumors (graphs, lower panels). CD31, platelet endothelial cell adhesion molecule 1; LAM, laminin. 

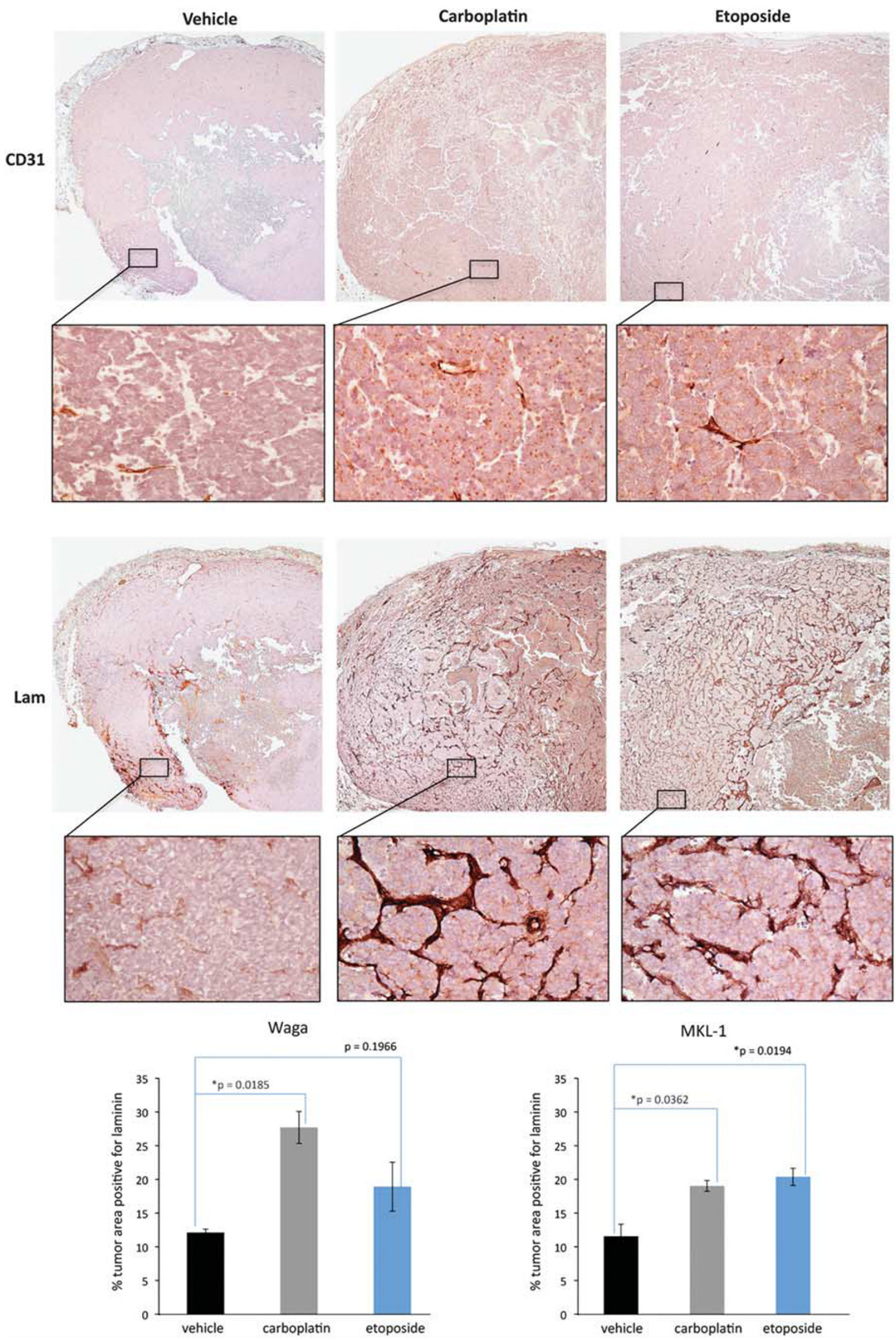

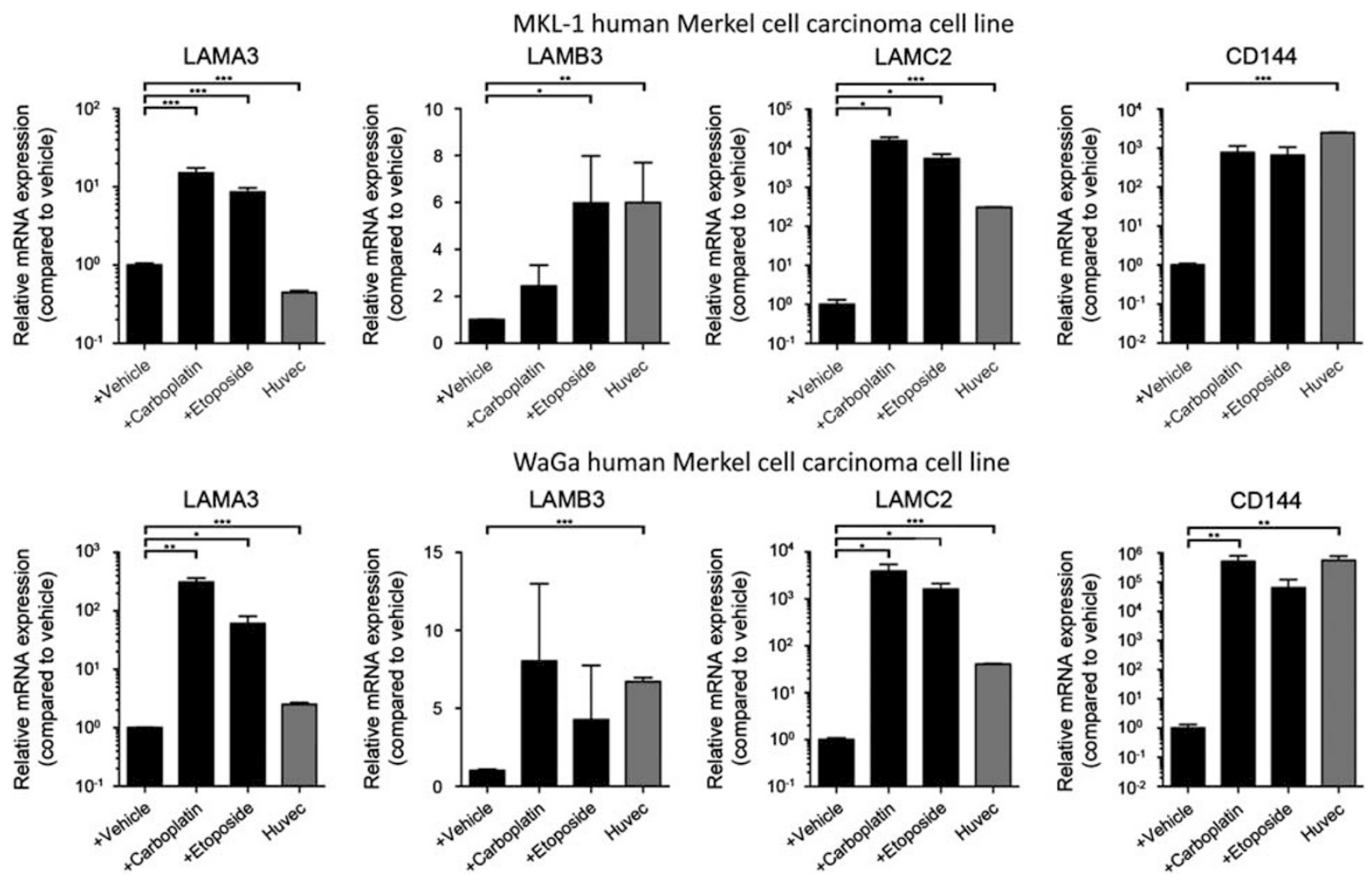

Figure 4. Expression of vasculogenic mimicry-associated markers by carboplatin- and etoposide-resistant Merkel cell carcinoma cells. Relative LAMA3 (left), LAMB3 (center, left), LAMC2 (center, right), and vascular endothelial-cadherin (CD144; right) mRNA expression (mean \pm s.e.m.) by carboplatinand etoposide-resistant vs vehicle-treated MKL-1 (top) and WaGa (bottom) cells, as determined by real-time quantitative reverse-transcription PCR. Established HUVEC served as a positive control. Data are representative of $n=3$ independent experiments. $\left({ }^{*} P<0.05,{ }^{* *} P<0.01,{ }^{* * *} P<0.001\right)$. CD144, vascular endothelial-cadherin; HUVEC, human umbilical vein endothelial cells; LAM, laminin.

lines compared with vehicle-treated controls, although statistical significance was reached only in carboplatin resistant WaGa cells (Figure 4).

\section{Patient MCC}

Seven MCC specimens from six patients were evaluated for the presence of anastomosing networks similar to those observed in MCC xenografts. Four of them showed similar, although less elaborate and dense, linear branching structures on PAS stain (Table 2); these were present diffusely throughout the tumor in one case and distributed more focally in the remaining three cases. Serial sections demonstrated identical patterns by laminin IHC for each of these four cases. Although $\mathrm{CD}_{3} 1^{+}$vessels in patient tumors were more abundant than in xenografts (average 30 cross-sectional $\mathrm{CD} 1^{+}$vessel profiles (range of $21-42$ ); $n=7$ ), they spatially coincided with only a fraction of the networks defined by laminin immunoreactivity in the four positive cases (Figure 5, upper and middle panels). Immunoreactivity for Ulex europaeus further confirmed the pattern of endothelial distribution detected by CD31 (data not shown). Computerassisted image analysis of sequential sections from one of these specimens confirmed that CD31 staining was asso- ciated with $20 \%$ of the intratumoral networks defined by laminin IHC. In addition, immunofluorescence dual labeling established these networks to be associated with positivity for CD144 but not for CD31. Interposed tumor vessels were positive for both epitopes (Figure 5, bottom panels). Taking all seven human MCC specimens as a group, no clear associations between presence or extent of VM and presumed lesion aggressiveness (that is, primary $v s$ recurrence vs metastasis) or chemotherapy treatment status were observed (Table 2).

\section{DISCUSSION}

VM is a mechanism intrinsic to a number of human cancers that is associated with aggressive behavior. ${ }^{6,12,19-32}$ Among skin cancers, melanoma and MCC are most virulent, and VM is a well-recognized phenomenon in melanoma. ${ }^{5,6,33,34} \mathrm{We}$ thus posited that VM may have a similar role in MCC. Because it has been found that VM is a more readily and consistently demonstrable phenomenon in conditions associated with intratumoral hypoxia, ${ }^{35}$ such as may occur in aggressive, rapidly growing tumors with high metabolic demands, ${ }^{36}$ we first employed a MCC xenograft model for experimental identification and manipulation of VM that, 
Table 2 Human MCC samples and VM

\begin{tabular}{|c|c|c|c|c|c|c|c|}
\hline Patient & Age & Specimen type & Chemotherapy & VM & Survival ${ }^{\mathrm{a}}$ & $\mathrm{T}^{\mathrm{b}}$ & Stage $^{b}$ \\
\hline 1 & 61 & Cutaneous primary & None & Focal & 2Y $1 \mathrm{~m} \mathrm{DOD}$ & $\mathrm{T} 2$ & $\| \mathrm{A}$ \\
\hline 2 & ND & LN metastasis & None & Focal & ND & ND & ND \\
\hline 3 & 83 & LN metastasis & None & Focal & $1 Y 10 \mathrm{~m}$ DOD & T1 & $\| \mathrm{A}$ \\
\hline 4 & 76 & LN metastasis & None & Absent & $8 m$ DOD & T4 & IIIB \\
\hline 5 & 77 & Cutaneous primary & None & Absent & $1 Y 7 m A$ & $\mathrm{~T} 4$ & IIIB \\
\hline 5 & 77 & Cutaneous recurrence $^{c}$ & Carbo + Eto & Absent & $1 Y 7 m A$ & $\mathrm{~T} 4$ & IIIB \\
\hline 6 & 78 & Cutaneous recurrence & Carbo + Eto & Diffuse & $3 Y 5 \mathrm{~m}$ DOD & $\mathrm{T} 1$ & IIIA \\
\hline
\end{tabular}

Abbreviations: A, alive; Carbo, carboplatin; DOD, died of the disease; Eto, etoposide; LN, lymph node; m, month(s); MCC, Merkel cell carcinoma; ND, no data; $\mathrm{T}$, tumor staging; VM, vasculogenic mimicry; $\mathrm{Y}$, year(s).

a Survival time from the date of initial diagnosis.

${ }^{\mathrm{b}}$ American Joint Committee on Cancer Staging System, 7th Edition.

'Same site.

like melanoma xenografts, exhibited accelerated growth rates as compared with patient tumors. Using two distinct human MCC lines, xenograft tumors remarkably similar to primary MCC in humans were generated. Initial PAS and laminin staining of these tumors revealed complex anastomosing and branching networks diffusely throughout tumor nodules. Importantly, the majority ( $>90 \%$ ) of these laminin-positive structures were unassociated with $\mathrm{CD} 31$ reactivity, thus exempting them from basement membranes integral to conventionally induced tumor angiogenesis. Regardless of CD31 negativity, laminin within tumors need not imply VM. We therefore utilized a panel of biomarkers associated with VM to further confirm our findings. These included CD144 (VE-cadherin), a marker associated with endothelial lineage and previously shown to be characteristic of $\mathrm{CD} 31^{-}$ tumor cells associated with laminin network formation; $;, 12$ VEGFR-1, a receptor involved in tumor angiogenesis and recently shown to promote and regulate laminin network formation in melanoma; ${ }^{5}$ and Nodal, an embryonic morphogen previously identified in association with VM both in experimental melanomas and patient tumors. ${ }^{17}$ These biomarkers further confirmed the presence of VM in MCC xenografts.

VM was originally considered by Hendrix and co-workers to be produced by less-differentiated populations of cancer cells capable of phenotypic and functional plasticity, ${ }^{6,7}$ and this insight is fortified by the association of melanoma subpopulations of established chemoresistance with laminin network formation regulated by the VEGFR-1 pathway. ${ }^{5}$ Because such subpopulations are notoriously resistant to chemotherapy and might be enriched in tumors as a consequence of chemotherapy, ${ }^{4,37,38}$ we hypothesized that VM driven by melanoma subpopulations ${ }^{5,39}$ may also be similarly protected or augmented in MCC. Indeed, our study indicates that laminin networks are enhanced in xenografts derived from two separate MCC lines in response to two different chemotherapeutic agents of established clinical relevance to the treatment of patient MCC, such as etoposide and carboplatin. Furthermore, we performed in vitro assays in the absence of endothelial cells that produce laminin in tumor xenografts or patient samples, and were able to demonstrate increased mRNA levels for three laminin isoforms and CD144 in etoposide- and carboplatinresistant WaGa and MKL-1 cells compared with vehicletreated counterparts. Although not all measurements were statistically significant, a trend consistent with enhanced VM was observed. These results support the notion that chemoresistance induces a VM-like phenotype in MCC lines in vitro. In spite of the limited numbers of samples evaluated in this study, taken together, these results suggest that MCC subpopulations capable of VM may have a survival advantage in certain therapeutic settings.

Although laminin networks are also detected in MCC from patients, they are not as well developed or elaborately expressed as in xenograft tumors. This difference could be related to differences in growth rates between naturally occurring and xenograft tumors. This potentially has at least two interrelated consequences: (1) robust tumor expansion in xenografts may outstrip the ability of stromal-derived murine angiogenesis to populate the growing nodule with authentic tumor vessels ${ }^{36}$ and (2) resultant production of a hypoxic, metabolically stressed microenvironment may drive VM. ${ }^{35}$ Indeed, $\mathrm{CD} 1^{+}$vessels are considerably fewer in xenografts than in patient tumors that generally have gradual growth over many months to years, a finding that supports this hypothesis. In 1986, Hall et al ${ }^{40}$ reported that human primary and secondary MCC $(n=9)$ showed no detectable laminin immunoreactivity, except for that associated with small vessels and epidermal basement membranes. MCC are recognized to be vascularized tumors capable of expressing angiogenic factors that promote their growth and for which targeted anti-angiogenic drugs have been proposed as a 

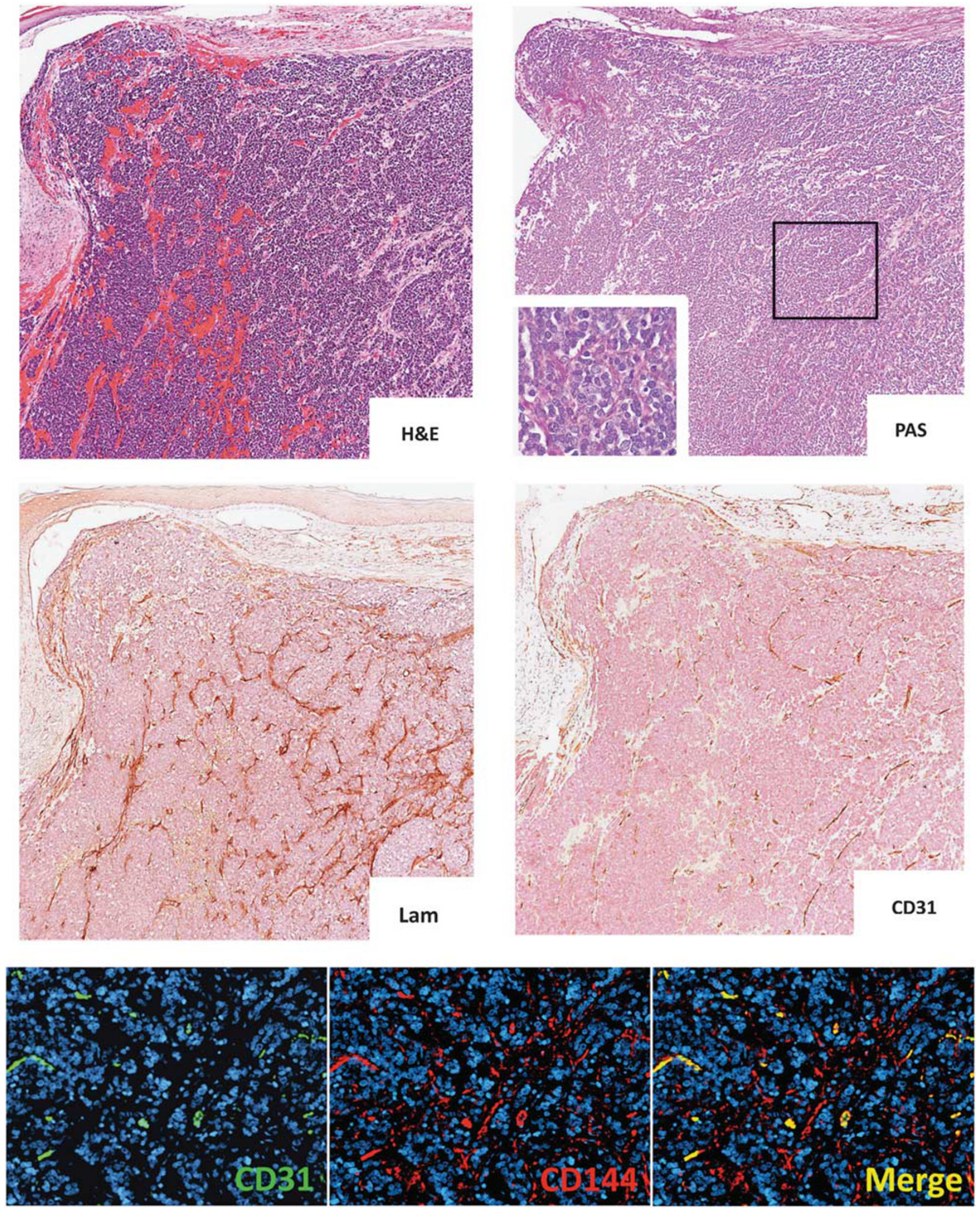

Figure 5. Vasculogenic mimicry in patient Merkel cell carcinoma. PAS-positive networks also are demonstrable by laminin immunohistochemistry, and a minority of these are associated with CD31 staining in adjacent sections (original magnification, $\times 100$; inset, $\times 1000$ ). Double immunofluorescence labeling for CD31 and vascular endothelial-cadherin (CD144) demonstrates an architectural pattern of CD144 positivity similar to that seen with laminin, and distinct from the comparatively few and discrete $\mathrm{CD} 31^{+}$vessels (original magnification, $\times 200$ ). CD31, platelet endothelial cell adhesion molecule 1; H\&E, hematoxylin and eosin; LAM, laminin; PAS, periodic acid-Schiff. 
therapeutic strategy. ${ }^{41}$ Thus, it is possible that intratumoral laminin immunoreactivity could have been entirely attributed to conventional angiogenesis, emphasizing the potential difficulty in recognizing VM networks without additional markers to prove the absence of endothelium. Alternatively, it is possible that differences in sensitivity of laminin detection are responsible for the conclusion in the Hall study.

Whether VM in MCC is involved solely in nutrient perfusion via laminin-lined sinusoidal conduits that accommodate extravasated blood from leaky tumor vessels, as has been posited ${ }^{6}$ remains unknown. An intriguing possibility is that VM may additionally provide a three-dimensional stimulatory scaffold that supports tumorigenic expansion of proliferating neoplastic cells. The recognized role of laminin as a cancer cell mitogen, ${ }^{42,43}$ as well as the propensity for cancer growth to exhibit stromal/extracellular matrix dependency, ${ }^{44-46}$ offers potential support to this theory. The establishment of VM in MCC evaluable in a xenograft model relevant to human disease now provides a pathway for additional research into these and other issues relating to elucidation of mechanisms of MCC virulence. In the present study, the results on a limited number of human specimens did not show a clear association between presence or extent of $\mathrm{VM}$ and lesion aggressiveness or response to chemotherapy. However, assessment of a larger cohort of annotated patient biospecimens will be necessary to determine whether $\mathrm{VM}$ is an informative biomarker for prognosis, staging, and determination of therapeutic resistance in MCC. Nonetheless, the establishment of VM in MCC, and data indicating its resistance to conventional chemotherapy, provides new insights into underlying pathways for tumor virulence that now may be further explored mechanistically.

\section{ACKNOWLEDGMENTS}

NIH-P50 CA93683 and R01 CA58467 (GFM) and Brigham and Women's Hospital Department of Dermatology Funding for Young Investigators (TS) supported the present work. TS is the recipient of a Research Career Development Award from the Dermatology Foundation.

\section{DISCLOSURE/CONFLICT OF INTEREST}

The authors declare no conflict of interest.

1. Akhtar S, Oza KK, Wright J. Merkel cell carcinoma: report of 10 cases and review of the literature. J Am Acad Dermatol 2000;43:755-767.

2. Yiengpruksawan A, Coit DG, Thaler HT, et al. Merkel cell carcinoma. Prognosis and management. Arch Surg 1991;126:1514-1519.

3. Laga AC, Lai CY, Zhan $\mathrm{Q}$, et al. Expression of the embryonic stem cell transcription factor SOX2 in human skin: relevance to melanocyte and merkel cell biology. Am J Pathol 2010;176:903-913.

4. Schatton T, Murphy GF, Frank NY, et al. Identification of cells initiating human melanomas. Nature 2008;451:345-349.

5. Frank NY, Schatton T, Kim S, et al. VEGFR-1 expressed by malignant melanoma-initiating cells is required for tumor growth. Cancer Res 2011;71:1474-1485.

6. Maniotis AJ, Folberg R, Hess A, et al. Vascular channel formation by human melanoma cells in vivo and in vitro: vasculogenic mimicry. Am J Pathol 1999;155:739-752.
7. Seftor EA, Meltzer PS, Kirschmann DA, et al. Molecular determinants of human uveal melanoma invasion and metastasis. Clin Exp Metastasis 2002:19:233-246.

8. Folkman J. Tumor angiogenesis: therapeutic implications. N Engl J Med 1971;285:1182-1186.

9. Hanahan D, Folkman J. Patterns and emerging mechanisms of the angiogenic switch during tumorigenesis. Cell 1996;86:353-364.

10. Fukumura $D$, Xavier $R$, Sugiura $T$, et al. Tumor induction of VEGF promoter activity in stromal cells. Cell 1998:94:715-725.

11. Bissell MJ.. Tumor plasticity allows vasculogenic mimicry, a novel form of angiogenic switch. A rose by any other name? Am J Pathol 1999:155:675-679.

12. Hendrix MJ, Seftor EA, Meltzer PS, et al. Expression and functional significance of VE-cadherin in aggressive human melanoma cells: role in vasculogenic mimicry. Proc Natl Acad Sci USA 2001;98:8018-8023.

13. Rodig SJ, Cheng J, Wardzala J, et al. Improved detection suggests all Merkel cell carcinomas harbor Merkel polyomavirus. J Clin Invest 2012;122:4645-4653.

14. Frank NY, Margaryan A, Huang $Y$, et al. ABCB5-mediated doxorubicin transport and chemoresistance in human malignant melanoma. Cancer Res 2005;65:4320-4333.

15. Lian CG, Xu Y, Ceol C, et al. Loss of 5-hydroxymethylcytosine is an epigenetic hallmark of melanoma. Cell 2012;150:1135-1146.

16. Fichtner I, Rolff J, Soong R, et al. Establishment of patient-derived nonsmall cell lung cancer xenografts as models for the identification of predictive biomarkers. Clin Cancer Res 2008;14:6456-6468.

17. McAllister JC, Zhan Q, Weishaupt C, et al. The embryonic morphogen, Nodal, is associated with channel-like structures in human malignant melanoma xenografts. J Cutan Pathol 2010;37(Suppl 1):19-25.

18. Wilson $B J$, Schatton $T$, Zhan $Q$, et al. ABCB5 identifies a therapyrefractory tumor cell population in colorectal cancer patients. Cancer Res 2011;71:5307-5316.

19. Shirakawa K, Kobayashi H, Heike $\mathrm{Y}$, et al. Hemodynamics in vasculogenic mimicry and angiogenesis of inflammatory breast cancer xenograft. Cancer Res 2002;62:560-566.

20. Wang R, Chadalavada K, Wilshire J, et al. Glioblastoma stem-like cells give rise to tumour endothelium. Nature 2010;468:829-833.

21. Sun B, Zhang S, Zhao $X$, et al. Vasculogenic mimicry is associated with poor survival in patients with mesothelial sarcomas and alveolar rhabdomyosarcomas. Int J Oncol 2004;25:1609-1614.

22. Hendrix MJ, Seftor EA, Kirschmann DA, et al. Molecular biology of breast cancer metastasis. Molecular expression of vascular markers by aggressive breast cancer cells. Breast Cancer Res 2000;2:417-422.

23. Liu R, Yang $\mathrm{K}$, Meng $\mathrm{C}$, et al. Vasculogenic mimicry is a marke of poor prognosis in prostate cancer. Cancer Biol Ther 2012;13: 527-533.

24. Chai DM, Bao ZQ, Hu JG, et al. Vasculogenic mimicry and aberrant expression of HIF-lalpha/E-cad are associated with worse prognosis of esophageal squamous cell carcinoma. J Huazhong Univ Sci Technolog Med Sci 2013;33:385-391.

25. Lu XS, Sun W, Ge CY, et al. Contribution of the PI3K/MMPs/ Ln-5gamma2 and EphA2/FAK/Paxillin signaling pathways to tumor growth and vasculogenic mimicry of gallbladder carcinomas. Int J Oncol 2013;42:2103-2115.

26. Wang SY, Ke YQ, Lu GH, et al. Vasculogenic mimicry is a prognostic factor for postoperative survival in patients with glioblastoma. J Neurooncol 2013;112:339-345.

27. Yu L, Wu SW, Zhou L, et al. Correlation between bacterial L-form infection, expression of HIF-1alpha/MMP-9 and vasculogenic mimicry in epithelial ovarian cancer. Sheng Li Xue Bao 2012;64:657-665.

28. Wu S, Yu L, Wang D, et al. Aberrant expression of CD133 in non-small cell lung cancer and its relationship to vasculogenic mimicry. BMC Cancer 2012;12:535.

29. Lin $P$, Wang $W$, Sun BC, et al. Vasculogenic mimicry is a key prognostic factor for laryngeal squamous cell carcinoma: a new pattern of blood supply. Chin Med J (Engl) 2012;125:3445-3449.

30. Sun $W$, Shen $Z Y$, Zhang $H$, et al. Overexpression of HIF-1alpha in primary gallbladder carcinoma and its relation to vasculogenic mimicry and unfavourable prognosis. Oncol Rep 2012;27:1990-2002.

31. Wang SY, Yu L, Ling GQ, et al. Vasculogenic mimicry and its clinical significance in medulloblastoma. Cancer Biol Ther 2012;13:341-348.

32. Baeten $\mathrm{Cl}$, Hillen $\mathrm{F}$, Pauwels $\mathrm{P}$, et al. Prognostic role of vasculogenic mimicry in colorectal cancer. Dis Colon Rectum 2009;52:2028-2035. 
33. van Beurden A, Schmitz RF, van Dijk CM, et al. Periodic acid Schiff loops and blood lakes associated with metastasis in cutaneous melanoma. Melanoma Res 2012;22:424-429.

34. Kirschmann DA, Seftor EA, Hardy KM, et al. Molecular pathways: vasculogenic mimicry in tumor cells: diagnostic and therapeutic implications. Clin Cancer Res 2012;18:2726-2732.

35. Sun B, Zhang D, Zhang $S$, et al. Hypoxia influences vasculogenic mimicry channel formation and tumor invasion-related protein expression in melanoma. Cancer Lett 2007;249:188-197.

36. Vaupel P, Kallinowski F, Okunieff P. Blood flow, oxygen and nutrient supply, and metabolic microenvironment of human tumors: a review. Cancer Res 1989;49:6449-6465.

37. Chartrain M, Riond J, Stennevin A, et al. Melanoma chemotherapy leads to the selection of ABCB5-expressing cells. PLoS One 2012 7:e36762.

38. Cheung ST, Cheung PF, Cheng CK, et al. Granulin-epithelin precursor and ATP-dependent binding cassette (ABC)B5 regulate liver cancer cell chemoresistance. Gastroenterology 2011;140:344-355.

39. Chiao MT, Yang YC, Cheng WY, et al. CD133 + glioblastoma stem-like cells induce vascular mimicry in vivo. Curr Neurovasc Res 2011;8: 210-219.

40. Hall PA, d'Ardenne AJ, Butler MG, et al. Cytokeratin and laminin immunostaining in the diagnosis of cutaneous neuro-endocrine (Merkel cell) tumours. Histopathology 1986;10:1179-1190.

41. Kukko H, Koljonen V, Lassus $P$, et al. Expression of vascular endothelial growth factor receptor-2 in Merkel cell carcinoma. Anticancer Res 2007;27:2587-2589.

42. Mortarini R, Gismondi A, Maggioni A, et al. Mitogenic activity of laminin on human melanoma and melanocytes: different signal requirements and role of beta 1 integrins. Cancer Res 1995;55:4702-4710.
43. Panayotou G, End $P$, Aumailley $M$, et al. Domains of laminin with growth-factor activity. Cell 1989;56:93-101.

44. Bremnes RM, Donnem T, Al-Saad S, et al. The role of tumor stroma in cancer progression and prognosis: emphasis on carcinoma-associated fibroblasts and non-small cell lung cancer. J Thorac Oncol 2011;6: 209-217.

45. Pouliot N, Kusuma N.. Laminin-511: a multi-functional adhesion protein regulating cell migration, tumor invasion and metastasis. Cell Adh Migr 2013;7:142-149.

46. Lu P, Weaver VM, Werb Z.. The extracellular matrix: a dynamic niche in cancer progression. J Cell Biol 2012;196:395-406.

47. Folberg R, Hendrix MJ, Maniotis AJ.. Vasculogenic mimicry and tumor angiogenesis. Am J Pathol 2000;156:361-381.

48. Shibuya M.. Differential roles of vascular endothelial growth facto receptor-1 and receptor-2 in angiogenesis. J Biochem Mol Biol 2006; 39:469-478.

49. Vartanian A, Stepanova E, Grigorieva I, et al. VEGFR1 and PKCalpha signaling control melanoma vasculogenic mimicry in a VEGFR2 kinaseindependent manner. Melanoma Res 2011;21:91-98.

50. Hendrix MJ, Seftor EA, Seftor RE, et al. Reprogramming metastatic tumour cells with embryonic microenvironments. Nat Rev Cancer 2007;7:246-255

51. Seftor RE, Seftor EA, Koshikawa N, et al. Cooperative interactions of laminin 5 gamma2 chain, matrix metalloproteinase-2, and membrane type-1-matrix/metalloproteinase are required for mimicry of embryonic vasculogenesis by aggressive melanoma. Cancer Res 2001; 61:6322-6327.

52. Simon-Assmann P, Orend G, Mammadova-Bach E, et al. Role of laminins in physiological and pathological angiogenesis. Int J Dev Biol 2011;55:455-465. 